\title{
Theophil Bonet (1620-1689) \\ und die grundsätzliche Bedeutung seines «Sepulchretum» in der Geschichte der Pathologischen Anatomie
}

Von Heinrich Buess, Basel

Selbst demjenigen, der sich nur wenig mit der Geschichte der Medizin befaßt hat, wird Theophil Bonet wenigstens dem Namen nach vertraut sein. Und auf die Frage nach der wichtigsten Leistung des Genfer Arztes wird die Antwort ohne weiteres lauten, daß in seinem Sepulchretum die erste Sammlung von pathologisch-anatomischen Beobachtungen vorliege. Die Begründung dieses Urteils wird jedoch sogar bei Fachleuten nicht so leicht zur Hand sein. Denn - so eigenartig dies erscheint - es fehlt bis heute an einer eingehenden biographischen und quellenkritischen Würdigung von medizinhistorischer Seite, wie denn überhaupt die Geschichte der Pathologischen Anatomie trotz manchen verdienstvollen Monographien über einzelne Zeitabschnitte und Fachgebiete keine umfassende, unmittelbar auf den wichtigsten Texten aufbauende Bearbeitung gefunden hat. Es dürfte noch lange dauern, bis das von Julius Hirschberg in der Geschichte der Augenheilkunde aufgestellte Vorbild für alle medizinischen Disziplinen erreicht sein wird. In biographischer Hinsicht hat die schöne, von dem spärlichen, in Neuenburg vorhandenen Archivmaterial ausgehende Studie des früheren dortigen Spitalarztes Eduard Cornaz (1825-1904) wenigstens einige weitere Aufschlüsse über die öffentliche Wirksamkeit BonEts gebracht.

Wenn auch die vorliegende Untersuchung ${ }^{1}$ in dieser Hinsicht enttäuschen wird, so sind ihr doch sämtliche heute zugänglichen handschriftlichen Dokumente zugrunde gelegt, und es soll versucht werden, das persönliche Bild des Genfer Arztes wenigstens in einen bescheidenen zeitgeschichtlichen Rahmen hineinzustellen. Was die ergographische Seite betrifft, so zieht unsere Skizze in bewußter Beschränkung lediglich die wichtigsten gynäkologischen Beobachtungen heran, um an ihnen die Arbeitsweise des Genfer Arztes zu illustrieren.

\footnotetext{
1 Es darf vielleicht angemerkt werden, daß die ersten Vorarbeiten über BoNET, die auf eine Anregung von Herrn Prof. PAul Diepgen zurückgehen, mehrere Jahre zurückliegen. Doch scheint in der Zwischenzeit keine einschlägige Arbeit erschienen zu sein.
} 


\section{Das Leben Theophil Bonets}

Um die Mitte des 16. Jahrhunderts erlebte Genf einen gewaltigen Aufschwung. Die von FAREL 1536 eingeführte Reformation hatte sich unter der straffen Zucht Johannes Calvins gefestigt. Aber nicht nur die Kirche, sondern, dem Humanisten Calvin entsprechend, auch das übrige kulturelle Leben der Rhonestadt wurde neu gestaltet. Im Jahre 1559 waren das «Collège» und die Akademie ins Leben gerufen worden. Von der Theologie aus wurde der Unterricht an der Akademie bald auf die ganze Philosophie und die Rechtslehre ausgedehnt. Viele der berühmtesten Ärzte und Naturforscher gehörten ihr später als Lehrer an.

Aber auch dadurch zog in Genf neues wissenschaftliches Leben ein, daß die von dort ausgehende neue Lehre eine große Zahl von Flüchtlingen aus den umliegenden Ländern in der beschützenden Rhonestadt Aufnahme suchen ließ. Die Wirkung dieser Gelehrten erstreckte sich allerdings mehr nach außen, indem die kleine Stadt beim Fehlen einer ärztlichen Fakultät dem Lehrtalent keine Möglichkeiten der Entfaltung zu bieten vermochte. So entstanden die Sammelwerke der beiden großen Genfer Ärzte des 17. Jahrhunderts, das Sepulchretum von Theophil Bonet und die Histoire de la médecine von Daniel Le Clerc (1652-1728), die beide zu den ersten groBen Darstellungen einer neuen medizinischen Disziplin gehören. Diese beiden Ärzte entstammen Familien, die im Laufe des 16. Jahrhunderts in Genf eingewandert sind.

Das Geschlecht der Bonet soll früher in Rom ansässig gewesen sein. Der Großvater von Theорніц B., zuerst Leibarzt des Herzogs von Savoyen in Turin, kam nach Lyon. Der Vater, ANDRé B. (gest. 1639), der im Jahre 1617 ins Bürgerrecht der Stadt Genf aufgenommen wurde, besaß ebenfalls den Ruf eines tüchtigen Praktikers. So folgten seine beiden Söhne aus zweiter Ehe, Jean (1615-1688) und Theоpнil, der Familientradition. Auch von den Nachkommen dieser beiden bedeutenden Genfer Ärzte ergriffen mehrere den ärztlichen Beruf, so daß es eine ganze Arztdynastie der Bonet gibt. Das überragende Glied der Familie ist Theоpнil B. Leider ist über sein Leben wenig bekannt. Weitere Bemühungen in dieser Richtung blieben sozusagen erfolglos. Vielleicht fördert weiteres Suchen mehr zutage ${ }^{2}$.

${ }^{2}$ Den Herren Drs. Geisendorf (Staats-Archiv Genf), André Bovet (Bibl. publ. Neuchâtel) und August Bouvier (Bibl. publ. et univ. Genf) sei für ihre liebenswürdigen Auskünfte auch hier bestens gedankt. 
Theophil Bonet wurde am 6. März 1620 in Genf geboren. Mit 23 Jahren soll er nach Studien an mehreren berühmten Universitäten zum Doktor promoviert worden sein.

Dank den bereits erwähnten Nachforschungen von CoRNAZ und einigen Angaben aus dem Staatsarchiv Genf ${ }^{3}$ läßt sich über die weitere Laufbahn B.s etwa folgendes sagen: Nach seiner Heimkehr ließ sich B. als Arzt in Genf nieder, vielleicht in der Praxis des kurz vorher gestorbenen Vaters. 1652 wurde er in den Rat der Zweihundert aufgenommen, hatte sich also durch sein praktisches Wirken wohl schon einiges Ansehen erworben. Ob er aus finanziellen Gründen oder, dem Ehrgeiz der damaligen Ärzte huldigend, sich nach einem festen Einkommen umsah, ist nicht bekannt. Jedenfalls folgte B., obschon er in Genf mindestens ein Haus besaß, im Jahre 1656 einem Ruf als Stadtarzt nach Neuenburg. Die Stelle hatte einige Jahre der Genfer Jean Sarasin (1610-1676) bekleidet, der B. vielleicht zu diesem Schritt ermunterte. Seine Familie ließ B. wohl in Genf zurück.

Seit 1592 waren die Herrschaft Valangin und die Grafschaft Neuenburg zum Fürstentum vereinigt. Dadurch hatte die alte Stadt neue Entwicklungsmöglichkeiten erhalten, die insbesondere das Bürgertum erstarken ließen. Mit dem Medizinalwesen war es allerdings schlecht bestellt, da die Bürger von Neuenburg nur Scherer und Apotheker stellten; die Ärzte indessen mußten von auswärts geholt werden. Seit dem Basler Aвraham BEcK (wohl einem Sohn des Humanisten PHILIPP BECK) hatten mehrere Fremde den Posten des Stadtarztes versehen. Der Stadtrat empfing B. mit hohen Ehren, vergütete ihm die Reisekosten reichlich und schenkte seinen Forderungen Gehör. Kaum ein Jahr später wurde B. vom regierenden Fürsten HeNri II. auch zum Leibarzt ernannt (7. August 1657). Für beide Stellen bezog B. ein ansehnliches Gehalt, als Stadtarzt hatte er Anrecht auf Wohnung und Garten, ein Maß Wein und Korn sowie zwei Batzen für einen behandelten Kranken.

B. war sich seiner Verantwortung bewußt. Als erfahrener Arzt sah er auch die Mißstände in der Neuenburger Gesundheitsordnung und suchte verbessernd einzugreifen. Schon ein Jahr nach seiner Ankunft (7. Oktober 1657) wurde eine neue Ordnung über die Berufs-Ausübung der Ärzte, Scherer (Chirurgen) und Apotheker in Kraft gesetzt. Der Stadtarzt besaß von

${ }^{3}$ Das Staatsarchiv Genf schrieb mir: «. . . nous vous communiquons que nous ne possédons sur Theophile Bonet aucun document personnel, genre correspondance, journal etc. En revanche sa vie à Genève, de 1658-1689, a laissé quelques traces dans les minutes de notaires ou dans les Registres du Conseil d'Etat.» 
Rechts wegen die Befugnis, die fremden Ärzte und Chirurgen vor ihrer Zulassung in der Stadt zu prüfen; auch die einheimischen Apotheker und Hebammen mußten vor ihm ein Examen ablegen. In Seuchenzeiten war er für die Durchführung geeigneter Maßnahmen verantwortlich. Nach dem Urteil von Convaz war B. der bedeutendste Stadtarzt, den Neuenburg je besaß.

Um so bedauerlicher ist es, daß er als Fremder der Cliquenwirtschaft und dem Neid der Neuenburger Patrizier zum Opfer fallen mußte. Schon zu Beginn seiner Tätigkeit hatte er wegen seines Anspruches auf einen Sitz in der Pfarrbank der Kirche Unannehmlichkeiten mit einem Geistlichen. Unter solchen "Vorpostengefechten» (Cornaz) hat B. wahrscheinlich auch die darauffolgenden Jahre zu leiden gehabt, obgleich er sich der besonderen Freundschaft des Staatskanzlers Georges de Montmollin (1628-1703) erfreute, dessen Haus er drei Jahre hindurch bewohnte und den er wegen einer hartnäckigen Trübung im rechten Auge behandelte(Briefe an J. C. BAUHIN $^{4}$ ).

Zum entscheidenden Zerwürfnis mit den Neuenburgern führte im Winter 1665 ein Angriff zweier angesehener Bürger der Stadt auf B.; es waren dies der junge Dr. Simon Chevalier, der die Stelle des Stadtarztes begehrte, und ein Apotheker. Wohl gewährten die Stadtbehörden die verlangte Genugtuung, indem die beiden Missetäter zu drei Tagen und drei Nächten Gefängnis verurteilt wurden. Der Bürgermeister sollte aber beim Fürsten ihre Begnadigung erwirken. Als diese tatsächlich ausgesprochen war, verließ B. im April 1666 die undankbare Stadt und kehrte nach Genf zurück. Seine fruchtbare Tätigkeit wurde ihm immerhin in einem anerkennenden Schreiben bestätigt.

Schon im Haus DE Montmollins hatte B. angefangen, Literatur zu sammeln für ein Buch, das er den praktischen Ärzten als «Vademecum» vermitteln wollte. Der Stoff dazu war den Schriften von Guillaume Baillou (1538-1616) (Ballonius) entnommen, einem Professor in Paris, der als nüchterner Beobachter für die praktische Medizin große Bedeutung besaß. In den Notariatsakten ist schon in der Neuenburgerzeit zweimal von der Vermietung seines Hauses in St-Germain, neben dem er offenbar ein zweites besaß, und von finanziellen Geschäften, auch mit seinen Verwandten

- Aufbewahrt in der Handschriftenabteilung der Universitätsbibliothek Basel (Sign. Mscr. G 2 I 10). Aus räumlichen Gründen wird hier nicht näher auf die interessante Krankengeschichte eingegangen, die dem berühmten Basler Praktiker vom jungen B. in extenso mitgeteilt wird, weil er von ihm Ratschläge für die Behandlung erwartete. 
die Rede 5 . Im Jahre 1669 erwarb B. eine Scheune und einen Stall mit einem kleinen Hof.

Seine Gattin, eine Tochter des damals bekannten Gelehrten FrIEdRICH Spanheim (1600-1649) aus der Pfalz, der 1625 als Professor der Philosophie nach Genf kam, fünf Jahre später Theologie an der Akademie lehrte und bis zur Würde des Rektors aufstieg (1635), schenkte ihm mehrere Söhne (André, Théophil, Fréderic, Louis Fréderic), von denen die beiden letzteren im diplomatischen Dienst bekannt wurden ${ }^{6}$. Die folgenden Jahre in Genf müssen in den häuslichen Verhältnissen B.s manche Trübung mit sich gebracht haben, so ist die Rede von einem Prozeß mit den Diakonen der «Bourse francaise» (20. Juni 1670), einer Stiftung zur Unterstützung von französischen Refugianten, und wenige Jahre später wird berichtet

${ }^{5}$ Es folgen hier die vom Staatsarchiv Genf mitgeteilten Auszüge aus den Notariatsakten während der Neuenburger Zeit:

1658, 19 mars. - Location de maison proche le temple de St-Germain pour fl. 600 p. an, par Spectable Robert Vaudenet, au nom de Spectable ThÉophil Bonet, docteur en médecine, citoyen, demeurant à Neufchastel, à JEAn RoBIN.

M. Pinault, not., vol. 46, f. 144

1660, 25 avril. - Obligation de fl. 8300 pour prest au $6 \% 3 \%$ du sieur THÉOP HILBONET à Jean Du Pan par l'entremise de Spectable Robert Vaudenet.

P. Jovenon, not., vol. 16, f. 575

1662, 4 septembre. - Obligation de fl. 5000 pour prêt au $6 \%$ due par Noble et Spectable ThÉOPHIL Bonet, docteur en médecine, demeurant à Neufchastel, à Noble JACOB DUPAN.

S. LENIEPS not., vol. 7, f. 108

1666, 3 janvier. - Location par Spectable ThÉophil Bonet, docteur médecin, à Noble MaXrmilien D'Yvoy, écuyer, d'une maison sise vers le temple de St-Germain.

Pierre Gautier, not., vol. 20, f.1

Aus dieser letzten Eintragung geht wohl deutlich hervor, daß B.s Rückkehr aus Neuenburg unvorhergesehen war, da er sonst nicht ein Vierteljahr vorher sein Haus in Genf vermietet hätte.

${ }^{6}$ FrÉDERIC, geb. 1652, bekannt auch als Numismatiker, wurde 1688 Mitglied des Rates der Zweihundert und war als Rat und Gesandter in England des Kurfürsten von Brandenburg tätig. - LouIs-FrÉDÉRIc, Dr. méd. et jur., 1670-1761, gehörte ebenfalls den Zweihundert an und trat später in die Dienste des Königs von Preußen (1723 in den Adelsstand erhoben, 1761 Geschäftsträger in London), der bekanntlich als einer der Erben im Jahre 1707 in den Besitz von Neuenburg gelangte. Hier mögen die Beziehungen B.s als einstiger Stadtarzt von Neuenburg eine Rolle gespielt haben. Wichtiger war indessen wohl der Umstand, daß Ezéchiel Spanheim (1629-1710) im Dienste des Großen Kurfürsten stand und später in London als preussischer Gesandter lebte. Dieser Schwager B.s ist in die Geschichte der Wissenschaften als einer der Gründer der Berliner Akademie eingegangen. 
über einen zwischen B. und seinen Söhnen ANDRÉ und THÉOPHIL zustandegekommenen Vergleich (1674), der gewisse «finanzielle Differenzen» zum Abschluß gebracht haben soll. An diesen Streitigkeiten waren offenbar sein Bruder Jean und sein Neffe ANDré beteiligt. Unter diesem Zwist scheint auch B.s wirtschaftliche Lage weiter gelitten zu haben; denn am 1. Januar 1676 ersuchte er mit der Begründung, daß er eine «zahlreiche Familie» habe und sich lange Zeit in Neuenburg aufgehalten habe, den Kleinen Rat um Befreiung von der «taxe des gardes». Dazu traten ernstliche gesundheitliche Störungen. Zu einer schon verhältnismäßig früh auftretenden Schwerhörigkeit, die ihn wohl immer mehr an das Studierzimmer band und die später zu völliger Taubheit führte, gesellte sich eine offenbar durch ein Herzleiden bedingte Wassersucht.

Den Eintragungen in den Ratsakten zufolge müßte aber B. doch ziemlich unerwartet gestorben $\operatorname{sein}^{7}$. So ist es möglich, daß er am 29. März 1689 einem Schlaganfall erlag. Mit Theophil B. verloren seine Mitbürger den ersten in der Reihe der berühmten Genfer Ärzte, der den Namen der Rhonestadt weithin bekanntwerden ließ.

\section{Die Stellung des «Sepulchretum» in der pathologisch-anatomischen Literatur}

Es kann nicht die Aufgabe der vorliegenden Studie sein, auf die Vorgeschichte der Pathologischen Anatomie einzugehen, die bekanntlich, soweit von eigentlichen medizinischen Beobachtungen die Rede ist, bis zum alexandrinischen Arzt Erasistratos (3.-2. Jahrhundert v. Chr.) zurückreichen. Nur auf eine Differenzierung in der historischen Betrachtung der Pathologie soll hingewiesen werden, die man m. E. nicht überall mit der nötigen Schärfe durchführt, obschon sie seit den rückblickenden Synthesen Rudolf Virchows immer wieder gefordert wurde (ich denke hier namentlich an seine Aufsätze zur Begründung der Zellularpathologie). Gemeint ist die Unterscheidung zwischen der wissenschaftlichen Beschäftigung mit dem Wesen der Krankheit, also den Spekulationen und Theorien der Krankheitsforschung, und den Untersuchungen über das Wesen der krankhaften Veränderung, die erst denkbar waren seit dem Eindringen des «anatomischen Gedankens» (VIRGHOw) in die abendländische Medizin. Die im Anschluß an Vesal mitgeteilten vereinzelten Beobachtungen der italie-

${ }^{7}$ So beschäftigte er sich noch im Februar-März mit Bauplänen, die allerdings etwas unklar sind. Das letzte Notariats-Protokoll (12. März) betrifft die Vermietung von zwei Etagen von möblierten Zimmern und Kellern in einem neben der «Arcade de la Cour de St-Pierre» gelegenen Haus. 
nischen Anatomen (Colombo, Eustackhi, Aranzio u. a.) leiten die Periode ein, die man als die Stufe der «groben Morphologie» bezeichnen kann. Deren Höhepunkt bilden die von Johannes SGhenk von Grafenderg (1530-1598) zusammengestellten Observationes medicae (Basel und Freiburg 1584-1597), in deren Titel schon das «insigne» dieses Zeitabschnittes

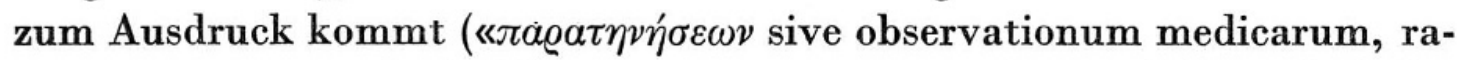
rarum, novarum, admirabilium et monstrosarum volumen»), liegt doch das Schwergewicht dieser rein «äußerlichen» Beobachtung (vgl. die Etymologie des griechischen Ausdrucks) auf den «seltenen und wunderbaren» Monstrosa, also auf den Mißbildungen, die allgemein als «lusus naturae» und Auswirkungen von dämonischen Mächten angesehen wurden.

Dieser ersten Stufe läßt sich nun die zweite als diejenige der «anatomischklinischen Kasuistik» gegenüberstellen. Und diese Stufe wird im wesentlichen durch das Sepulchretum von B. repräsentiert. Damit sind wir bei dem Werk unseres Genfer Arztes angelangt, das in seiner grundsätzlichen Bedeutung zu betrachten ist. Dabei wird zweckmäßigerweise von den im Vorwort niedergelegten allgemeinen Bemerkungen B.s auszugehen sein.

Was die Literatur über die krankhaften Veränderungen im 17. Jahrhundert betrifft, so hatten mit dem Aufschwung der von Frankreich ausgehenden Chirurgie und unter dem nachhaltigen Einfluß der Entdeckung Harveys die Studien über die Krankheitsprozesse eine andere Zielrichtung erhalten. Neben den verschiedenen, vom neuorientierten physiologischen Denken inspirierten Monographien über Kreislaufstörungen und die Folgen von Vergiftungen (hier sind vor allem die Arbeiten des Schaffhausers WePfer zu nennen) gehören zu dieser Richtung die zahlreichen «Spicilegia» der Chirurgen jener Zeit (Kerckring, BLANKaARD), in denen jedoch anatomische und pathologische Befunde noch miteinander vermengt sind.

a) Die Gestaltung

Das erste Werk, das ausschließlich der Darstellung pathologischer Leichenbefunde diente, war das Sepulchretum sive anatomia practica, ex cadaveribus morbo denatis, das in erster einbändiger Auflage im Jahre 1679 in Genf erschien. Es ist dies die von uns benützte Ausgabe. Ein zweitesmal wurde das Werk im Jahre 1700 vom bekannten Enzyklopädisten JoH. JAKоB MANGeT (1652-1742), dem jüngsten im Triumvirat hervorragender Genfer Ärzte in der zweiten Hälfte des 17. Jahrhunderts, herausgegeben. Diese zweite Ausgabe, die übrigens so wenig wie die erste frei ist von Fehlern (falsche Titel usw.), bringt Umstellungen von einzelnen Paragraphen, 
es sind eine Anzahl Anmerkungen weggelassen, der Druck weist einen neuen Satz auf, im übrigen liegt aber eine unveränderte Neuausgabe vor, die, soweit eine summarische Prüfung ergibt, kein besonderes Eingehen erfordert. Der Titel des Werkes, als «Begräbnisplatz» zu übersetzen (abgeleitet von sepulto, Intensivum von sepelio), entspricht der etwas umständlich-schwülstigen Schreibweise jener Zeit; im Untertitel kommt der Zweck zum Ausdruck, der erste Terminus für das, was wir heute pathologische Anatomie nennen.

Das Werk ist Herzog Friedrich August von Württemberg, Graf von Montbéliard und Eberstein, gewidmet, der einer Nebenlinie angehörte und geschichtlich ohne Bedeutung war. Er hatte B. auf der Durchreise durch Genf mehrmals als Patient aufgesucht. Der Herzog habe sicher Interesse an diesem «kleinen Geschenk mit den neuen Dingen», habe er doch in Deutschland «die schönste Bibliothek mit den zahlreichsten und am besten geordneten Büchern» errichtet. Dann folgen, einer Sitte der damaligen Zeit entsprechend, die Briefe vieler Ärzte, die B. zu seinem Unternehmen beglückwünschten. Besonders aufmunternd scheinen die Briefe von Тноmas Bartholin (1616-1680) und Joh. Conrad Peyer (1653-1712) gewesen zu sein, wie B. später im Vorwort hervorhebt.

Was die Form des Werkes betrifft, so sei kurz auf die Anordnung des Stoffes hingewiesen. Es wird zunächst, entsprechend dem alten Brauch, nach Körperregionen unterschieden (Kopf usw.), dann bilden innerhalb dieser Hauptteile einzelne hervorstechende Symptome (Schmerz, Tumor, plötzlicher Tod usw.) ein weiteres Einteilungsprinzip. Hier liegt also bereits eine allgemein-pathologische Idee zugrunde, ähnlich, wie sie vor B. schon bei Felix Platter (1536-1614), übrigens einem seiner wichtigsten Gewährsmänner, anzutreffen ist. In einem dritten Teil sind anhangsweise spezielle Kapitel behandelt (Fieber, Tumoren, Wunden, Frakturen, Luxationen, Gelenkerkrankungen, Hautleiden usw.), die größtenteils etwa unter dem modernen Sammelbegriff "pathologie externe» zu vereinigen sind. Im ganzen Werk sind 2934. Beobachtungen von 470 Autoren verarbeitet, eine gewaltige Zahl, die ungefähr die Größe der Leistung ermessen läßt.

\section{b) Die Zielsetzung}

Welche Aufgaben B. sich bei der Redaktion seines Sepulchretum stellte, geht klar hervor aus dem anfänglich etwas weitschweifigen Vorwort. «Die Anatomie der an Krankheiten Verstorbenen gibt uns die Möglichkeit, verborgene Ursachen ans Tageslicht zu ziehen und so den drohenden Tod 
verhüten zu können.» Er führe den Leser auf «einen abwegigen Pfad, der bisher nie begangen wurde» und dessen Begehung deshalb an «Verwegenheit» grenze; es wäre ihm recht gewesen, wenn ein anderer diese Arbeit übernommen hätte, statt daß er sie seinen unvorbereiteten Schultern habe zutrauen müssen. B. zählt dann seine Vorbilder auf von Petrus CAstellus (Castelli, 16. Jahrhundert) in Rom bis zu Ballonius und Daniel SenNERT (1572-1637), aus dem er hauptsächlich die «Ätiologie» geschöpft habe. Trotz allen diesen Vorarbeiten habe bisher ein nach dem Grundsatz «a capite ad calcem» durchgeführtes Werk gefehlt; dabei hält B. mit seiner Bewunderung vor den anatomischen Vorarbeiten nicht zurück. Es geht ihm nicht darum, die dem Kranken und seiner Umgebung eindrucksvollen Erscheinungen darzustellen, auch nicht darum, «das Außerordentliche der verborgenen Krankheiten» an den Tag zu bringen. B.s Ziel ist ein ganz schlichtes, nüchternes, dem alles Sensationelle, «Mirakulöse» abgeht, nämlich dies, «diejenigen Krankheiten», «die ihren bestimmten Sitz im Körper haben» und sich an den betreffenden Orten durch bestimmte Symptome äußern, abzuhandeln. Das bisherige Vorgehen, bei dem man das Schwergewicht auf die hervorstechenden Symptome oder das Überraschungsmoment legte, soll verlassen werden zugunsten des neuen Verfahrens, das ebenfalls auf den Praktiker ausgerichtet ist und ihm helfen soll, das Fundament für die Diagnose zu errichten: «Die anatomische Beobachtung ist Stoff und Grundlage der Praxis, was aus diesem Werk gelernt werden kann.»

B. will also seinen Kollegen das Hilfsmittel zur Verfügung stellen, anhand dessen er in der Beurteilung der Krankheiten zu einem sicheren, auf der anatomischen Veränderung begründeten Schluß gelangt. Wenn auch manche Ansätze in dieser Hinsicht schon vor ihm bestehen, so dürfte doch der Genfer Arzt zum erstenmal eine allgemeine Formulierung für dieses neue Ziel, die Anatomie in den Dienst der Krankheitsdiagnostik zu stellen, gegeben haben. Es darf also wohl schon hier festgestellt werden, daß B. nicht der ausschließliche Kompilator ist, als der er bisher vorwiegend galt, sondern daß er wenigstens in theoretisch-literarischer Hinsicht bereits den «anatomischen Grundgedanken» klar zum Ausdruck gebracht hat.

Doch ist B. doch nicht so ausschließlicher Büchergelehrter, daß er nicht wenigstens einige allgemeine Schlüsse aus seinem gedruckten und handschriftlichen Material gezogen hätte. Zwar läßt das Vorwort eine sachgemäße Gliederung vermissen, aber einige spezielle Hinweise auf praktische Fragen, die heute noch den Forscher am Sektionstisch beschäftigen, finden sich doch. So macht B. nachdrücklich auf einen Fehlschuß aufmerk- 
sam, der früheren Beobachtern häufig unterlaufen sei: «Auch dies ist festzuhalten hinsichtlich der Angaben über die Ursachen der Krankheiten: obschon ich meistens die Ableitung der Affektion nach Eröffnung der Leiche zu geben scheine, so dürften doch die angegebenen Ursachen dafür nicht immer adäquat sein, und zwar wegen verschiedener Veränderungen, die erst nach Erlöschen der Körperwärme oder kurz vor dem Exitus einzutreten pflegen.» Wenn B. hier auch an Äußerungen anknüpft, die als Auswirkungen vitalistischer Anschauungen in ähnlicher Form schon früher getan worden waren - er selbst zitiert den Satz des CeLSus: «Nichts ist törichter als zu glauben, wie etwas am Lebenden sei, gleichermaßen sei es auch am Toten» - so ist mit derselben prägnanten Klarheit m. W. vorher noch nie auf die prä- und postmortalen Erscheinungen an der Leiche aufmerksam gemacht worden. Als eindrückliches Beispiel erwähnt B. die Lunge, bei der man den Unterschied zwischen dem Zustand des Organs mühelos feststellen könne, je nachdem ob man unmittelbar nach dem Tod oder später seziere. Auch die Leber verändere sich, wie Theodor Kerckring (1640-1693) an während des Rennens verendeten Pferden beobachtet habe, nach dem Tode sehr schnell.

Es braucht große Erfahrung, um diese allgemeinen postmortalen Veränderungen von denjenigen zu unterscheiden, die durch eine Krankheit hervorgerufen werden. Dies ist offenbar der unausgesprochene Gedanke B.s, der ihn veranlaßt, einen höchst wichtigen Satz aus dem Buch De rhachitide (London 1660) von Francis Glisson (1597-1677) an dieser Stelle - ziemlich unvermittelt - einzuschalten: «Jede Krankheit hat ihre Eigenart an der Leiche, wo diese Eigenart fehlt, handelt es sich auch nicht um diese Krankheit.» Nur die sorgfältige Untersuchung könne, wie Guisson am Beispiel der «Gibbositas» gezeigt habe, vor Verwechslung und Täuschung bewahren. Auch hier wieder handelt es sich um eine der fundamentalen Erkenntnisse, die in dieser beiläufigen Form von B. seiner Sammlung vorangestellt wurde.

Nach kurzer Erwähnung der Entdeckung HaRveys und der an ihn sich anschließenden «neueren Werke» spricht der Genfer Arzt das Hauptverdienst am Sepulchretum in treuherziger Bescheidenheit seinen Zeitgenossen zu. Er habe nun das Material zusammengetragen, und auf diesem Fundament könne unter der Anleitung «eines geeigneten Architekten» der Bau in raschem Fortschreiten von unten bis oben hinaus errichtet werden. «Ich habe nur fruchtbares Material hinzugetragen, vielleicht mageres», werde man sagen, aber «kein Werk ist von Anfang an vollkommen», es liegt nach 
B. wenigstens «ein Anfangsgrund vor», «eine öde, rohe und ungeordnete Masse ..., eine Sandwüste ohne Stein, ein Ungeheuer, in das Licht hineingebracht wurde durch mich.» Schließlich beschwört B. die Leser seines Buches, daß es nur wahre, wirkliche Beobachtungen enthalte, für deren Glaubwürdigkeit die Namen vieler hervorragender Forscher Gewähr biete; und allfällige Fehler bittet er damit zu entschuldigen, daß das Werk während vieler Jahre in mühsamer Arbeit entstanden sei, ohne daß er vom «Privileg der Professoren» habe Gebrauch machen können, «die Kandidaten als Sekretären» manche Arbeit zuweisen könnten. Das Werk werde verschieden beurteilt werden, aber die Zustimmung werde auch nicht ausbleiben, und es würde andere dazu anspornen, Besseres zu leisten.

So weit das Vorwort des unermüdlichen Arztes, das wir in der von ihm gewählten Form - unter Weglassung der zeitbedingten weitschweifigen Stellen - wiedergeben wollten, um dem heutigen Interpreten einen möglichst wirklichkeitsnahen Eindruck zu vermitteln von seiner Persönlichkeit und vom zeitlichen Druck, aus dem heraus er sich die Stunden stehlen mußte, um wenigstens das literarische Gerippe zu schaffen, das andere nach ihm mit Fleisch und Blut zu füllen berufen sein sollten. Es dürfte deutlich geworden sein, daß B. von den Aufgaben der Leichenuntersuchung schon ganz klare Vorstellungen hatte. Diese wichtigen Ziele in einigen, hastig hingeworfenen Sätzen den an der medizinischen Forschung interessierten Zeitgenossen eingehämmert zu haben und damit für den «anatomischen Gedanken» in der Krankheitsforschung die erste Bresche geschlagen zu haben, darf ohne Zweifel als sein Verdienst bezeichnet werden. B. brach mit dem bisherigen «System» der Nosologien, Namen, Wesen, Ursachen und in die Augen springende Symptome in den Vordergrund zu rücken, und setzte an deren Stelle den Grundsatz, bei jeder Krankheit nach ihrem Sitz zu fragen, der Einheit von Krankheitsvorgang und anatomischer Veränderung nachzugehen und damit der ärztlichen Diagnostik das solide morphologische Fundament zu geben.

Wie wirkte sich nun diese allgemeine Zielsetzung im einzelnen aus? Von der Verwirklichung der im Vorwort skizzierten Absicht mußte der Erfolg seines mühseligen Unternehmens letzten Endes abhängen. Daß B. seinem Prinzip auch in der konkreten Durchführung treu blieb, dürften einige aus den gynäkologischen Kapiteln herausgegriffene Beispiele zeigen, die allerdings, was die Beobachtung als solche betrifft, auf das Konto der jeweiligen Autoren gehen, damit aber ein ausgezeichnetes Bild vom Stand der pathologisch-anatomischen Forschung des 17. Jahrhunderts bieten. 


\section{Die Verwirklichung des «anatomischen Prinzips» in der Gynäkologie des 17. Jahrhunderts}

Wie bereits früher bemerkt, ist das Sepulchretum nach dem alten topographischen Schema «a capite ad calcem» gegliedert. In den Abschnitten, die spezielle Organsysteme betreffen, werden die einzelnen Krankengeschichten bald nach dem Organ, in dem sich der Krankheitsprozeß abspielt, bald nach dem leitenden klinischen Symptom (siehe unten) eingeordnet. Die vorliegende skizzenhafte Übersicht wurde nach Organen in der Art eines modernen Lehrbuches unterteilt. Im ganzen bietet sich ein etwas monotones Bild, indem lediglich die groben morphologischen Veränderungen (Geschwülste, Mißbildungen, Lage-Anomalien usw.) erfaßt sind, wie dies auf der frühen Stufe der Forschung nicht anders erwartet werden darf. In unserem Zusammenhang werden wir auf die oft langfädigen Erörterungen über Descensus und Prolaps nicht eingehen, auch die im 17. Jahrhundert höchst aktuelle Diskussion über die Natur des Hymens und die damit zusammenhängenden klinischen Fragen seien übergangen.

Für die frühere gynäkologische Literatur sei auf die Werke von RoвY Kossmann $^{8}$ und Isidor Fischer ${ }^{9}$ hingewiesen, aus denen sich ergibt, daß in der alten Medizin bis zur Renaissance die Störungen der Menstruation ${ }^{10}$ in symptomatologischer Hinsicht im Vordergrund stehen und daß erst seitdem auch den morphologischen Abweichungen mehr Aufmerksamkeit geschenkt wurde.

a) Vulva und Scheide

Neben einem offenbar gestielten Fibroma pendulum, das von Georg PALMA $^{11}$ beschrieben wurde (p. 1140), ist unter den von der Vulva ausgehenden Tumoren eine livide, schwärzliche, blutig-eitrige, mit der umgebenden Haut stark verwachsene Geschwulst aufzuführen, über die in den Observationes medicae (lib. 3, cap. 34, Amsterdam 1641) des durch das Gemälde von Rembrandt bekanntgewordenen, vielseitigen Holländers NIcolaus TulP (1593-1674) berichtet wird. Über Metastasen (vgl. unten) findet sich nichts, nach dem Aussehen könnte es sich um ein verjauchtes

${ }^{8}$ R. Kossmann, Allgemeine Gynäkologie, 1. Theil: Geschichte der Gynäkologie, Berlin 1903.

${ }^{9}$ Geschichte der Gynäkologie, in: Halban-Seitz, Biologie und Pathologie des Weibes, Bd. 1, Berlin 1924.

${ }^{10}$ Vgl. dazu: Hans Georg Müller-Hess: Die Lehre von der Menstruation vom Beginn der Neuzeit bis zur Begründung der Zellenlehre, Abh. Gesch. Med. 27, Berlin 1938.

11 Über diesen Autor konnten keine genaueren Angaben ermittelt werden. 
Chorionepitheliom gehandelt haben, ohne daß man allerdings von einem Zusammenhang mit einer Gravidität erfährt. Ein Vergleich mit dem Original zeigt die wörtliche Übereinstimmung mit dem Text von B.

Tiefer in die anatomisch-topographischen Zusammenhänge führt ein Fall von ulzeriertem Scheidenkrebs (pag. 1354), den GerHard Blaes (erste Hälfte des 17. Jahrhunderts) aus Amsterdam im Jahre 1667 veröffentlichte; ohne daß im einzelnen die pathologisch-anatomische Eigenart dieses Tumors ersichtlich ist, fällt auf, daß dem Beobachter die Folgen der Harnröhrenstenose (Ureteren und Nieren-Dilatation) eindrücklich waren. BoNET bringt diesen Bericht, entsprechend dem wichtigsten Symptom, im Kapitel über den «Fluor», was die starke Konzession an die frühere Art der Darstellung zeigt, bei der das Schwergewicht auf dem klinischen Bild lag.

$\mathrm{Da}$ ß der Genfer Gelehrte auch die Früchte seiner umfangreichen Korrespondenz heranzog, zeigt der Bericht über einen Fall von Hämatometra, den er einer persönlichen Mitteilung des Basler Professors Johann Jakoв Harder (1656-1711) verdankte (28. Mai 1678) : «Anatomie einer nie menstruirten Jungfrau, deren Uterus von Natur verschlossen war.» Leider fehlt ein genauer Lokalbefund, vielmehr werden an diesem Bericht langatmige Erörterungen über die fehlende Gärung des Blutes als Krankheitsursache angeschlossen, wobei HARDER resigniert seine Unfähigkeit eingesteht, die Natur der gärenden Substanz zu eruieren.

\section{b) Gebärmutter}

Im Abschnitt «De hypogastrii dolore» (p. 1194, obs. 11) gibt B. einen Sektionsbefund wieder, der aus den 1611 in Genf erschienenen Observationes (Centuria II) des Fabricius Hildanus (1560-1634) stammt und im Jahre 1586 mit dem Chirurgen Jacques Aubert und Dr. Vindon ${ }^{12}$ in Lausanne erhoben wurde. Der Befund ist in summarischer Form festgehalten, was für die Annahme spricht, daß Fabricius diese Beobachtung später aus dem Gedächtnis niederschrieb.

Welche Fortschritte das 17. Jahrhundert in der anatomischen Beobachtung brachte, zeigt ein zweiter, im Kapitel über den «weiblichen Fluor, Ausscheidung nach der Geburt aus dem Uterus, Molen» (Lib. III, sect. 36, p. 1353) wiedergegebener ganz ähnlicher Krankheitsfall. Unter den klinischen Symptomen sind hervorgehoben:

12 Fehlt bei Eugène Olivier, Médicine et santé dans le pays Vaud, Bd. 2, Lausanne 1939. 
Fluor wegen exulzeriertem Uterus, vereiterte Leisten- und Uterus-Drüsen und Wiederauftreten unregelmäßiger Blutungen («inordinate») nach Aufhören der Menses seit dem 50. Lebensjahr. Der Tod war nach «Unterdrückung des Urins» (Urämie?) und galligem Erbrechen eingetreten. Der pathologisch-anatomische Befund zeigt erstaunliche Feinheiten, die eine minutiöse Untersuchungstechnik voraussetzen. Er sei nur auszugsweise wiedergegeben:

Leistendrüsen miteinander verbacken, Lymphgefäße gedehnt; das Ulcus der Gebärmutter greift über die Grenzen des Uterus hinaus auf die Vagina über («promoerium» im Original; entsprechend dem zweiten Stadium nach der internationalen Vereinbarung der Hygiene-Organisation des Völkerbundes von 1929); als Nebenbefund, der zeigt, wie gewissenhaft auch außerhalb des eigentlichen Krankheitssitzes gesucht wurde, ergab sich eine linke Niere «mit verdoppeltem Becken» (Mißbildung ?, Dilatation?).

Dieser ausgezeichnete Bericht wurde von B. aus den Briefen des Zürcher Arztes Johannes von Muralt (1645-1733) an JoH. Conrad Peyer in Schaffhausen entnommen, eines Forschers, dessen hervorragende Eigenschaften als Biologe erst seit der schönen Monographie Bernhard Peyers ${ }^{13}$ besser bekanntgeworden sind. Die hier wiedergegebene Stelle aus den Briefen von Muralts an Peyer, die offenbar von diesem eifrigen Mitarbeiter ${ }^{14}$ B.s für das Sepulchretum übermittelt worden war, beleuchtet eine bisher noch kaum bekannte Seite der Forschung des Zürcher Arztes, die offenbar ganz von seiner anatomischen Arbeit her zu verstehen ist. Und gerade hier scheint uns der «anatomische Gedanke» in der Pathologie des 17. Jahrhunderts einen ihrer Höhepunkte zu erreichen. Mit sicherem Blick greift B. gerade diese Einzelheit aus der Fülle seines Materials heraus, um sie als hell glänzendes Teilchen seinem großen Mosaik einzufügen. Deshalb schien sich ein ausführlicheres Eingehen auf diesen wohl nur in Handschrift vorliegenden Beitrag von Muralts zu lohnen.

Von den krankhaften Veränderungen des Corpus uteri, die B. aufgenommen hat, sei lediglich auf die Beschreibung eines Falles von Sarkom eingegangen, die der bereits genannte wichtige Gegner der scholastischen Medizin, Guillaume Baillou (Ballonius) wohl seinem Buch über die Frauenkrankheiten ${ }^{15}$ beigefügt hat. Daß der Doyen der Pariser Ärzte auch ein

13 Bernhard Peyer, Die biologischen Arbeiten des Arztes Johannes von Muralt (1645 bis 1733), Thayngen 1946.

14 Vgl. dazu auch C. Brunner und W. von Muralt, Aus den Briefen hervorragender Schweizer Arzte, Basel 1919, S. 19, wo BonEts Verdienste gegenüber dem abfälligen Urteil Hirschs von CoNrad BrUNNER mit Recht in Schutz genommen werden.

${ }^{15}$ De virginum et mulieris morbis, Paris 1643. Das Werk fehlt in Basel, so daß auf einen Vergleich des BoNETschen Textes mit dem Original verzichtet wurde. 
guter Beobachter war, dürfte aus dem nachstehenden Krankenbericht hervorgehen ${ }^{16}$ :

So wenig der lokale Sektionsbefund heute befriedigen kann, weil die Auswirkungen auf den Gesamtorganismus nicht berücksichtigt sind, so zeichnet sich die Beschreibung der Veränderung am Uterus durch ihre Genauigkeit und insbesondere durch ihre Maßangaben aus, womit eine der Forderungen der späteren Wissenschaft (MorgagNI, Virchow) hier bereits verwirklicht war. Gewiß fehlt noch jeglicher Hinweise auf die feinere Struktur dieses Gebildes, aber doch sind die Beziehungen zum Organ wenigstens andeutungsweise beschrieben.

Es handelt sich um eine kleingebaute Frau, die sich wegen Schwellung des Unterleibes für schwanger hielt und bald darauf an Marasmus zugrunde ging. Der Uterus war nach allen Seiten aufgetrieben (nach der «Wölbung», wie nach «Tiefe, Dicke und Weite»), die Wandung 5 Finger dick, nicht eindrückbar, mit der Hälfte 10 Pfund schwer, im Hohlraum eine fleischige Masse, die mit der Innenfläche zäh verwachsen war und den Uterus nach unten abschloß. BALlonius schnitt dieses merkwürdige Präparat zur Demonstration heraus, was umso mehr begründet war, als sich der offenbar maligne Tumor in einer mißgebildeten Gebärmutter (Uterus bicornis) befand.

\section{c) Eileiter}

Von größtem Interesse sind in der Pathologie der Tuben die Beobachtungen über Eileiterschwangerschaften, die B. mit ausführlichen theoretischen Erörterungen umkleidet. Es seien hier nur zwei Fälle herausgegriffen, von denen der zweite, weniger bekannte schon früher ${ }^{17}$ erwähnt wurde.

Die von JEAN Riolan d. J. (1580-1657) erzählte Krankengeschichte ${ }^{18}$ dürfte von der klinischen Seite her bereits bekannt sein, so da 3 auf sie nicht eingegangen zu werden braucht. Pathologisch-anatomisch ist von Interesse, daß der Sitz der Blutung im Uterushorn (nach damaliger Auffassung also in der Tube) gefunden wurde. Die Substanz um den 5 Finger langen Fetus war zerfetzt, auseinandergerissen; der angerissene Ast der Arterie wird vom Berichterstatter (Dr. Mercer, Bourges) bis zum rechten Horn des Uterus verfolgt, der selber leer und «wie bei einer Nichtgraviden beschaffen» war. Als Verbindung vom Uterus zum rechten Horn konnte trotz langem Suchen kein anderer Weg als der natürliche gefunden werden.

Auf die geschichtlichen Zusammenhänge wurde bereits früher (l.c.) hingewiesen, hier sei nochmals betont, daß der brandenburgische Leibarzt

${ }^{16}$ Kapitel «De ventris tumore», obs. 55, p. 1133 ss. In diesem Abschnitt sind mehrere Sektionsbefunde von Uterus myomatosus enthalten. Im obenstehenden Falle dürfte es sich um ein Sarkom handeln.

${ }^{17}$ Zur Entdeckung der tubaren Konzeption, Schweiz. med. Wschr. 76, 66 (1946).

${ }^{18}$ Sie ist seiner Anthropographia, Paris 1626, entnommen. 
Johann Sigmund Elsholtz (1623-1688) schon 1669 bei einem in Paris beobachteten Fall von Tubargravidität die Konzeption in den «Tuben», also nicht im «Uterushorn», annahm und damit in diesem Stück der klassischen Monographie Reinier DE GraAfs (1641-1673) vorgriff. Auch diese beiden Krankengeschichten lassen deutlich erkennen, bis zu welchen Feinheiten der Krankheitsforschung die anatomische Methode ausgebaut wurde. Darüber hinaus findet sich bei ElSHOLTz eine ausgesprochene physiologische Deduktion, wie sie durch HARveY in die Biologie eingeführt worden war. So gelangte ELsholtz offenbar von rein theoretischen Erwägungen ausgehend zur Annahme, daß im vorliegenden Fall die Vereinigung des weiblichen Samens oder Eies mit dem männlichen Samen in der Tube erfolgt sei, daß aber dieses «animatum» in der Mitte des Weges wegen eines Hindernisses hängengeblieben sei. Diese Beobachtung bilde, fährt ELsноLTz fort, einen einzigartigen Beweis für die Tatsache, daß keine Konzeption erfolge, es sei denn vorher etwas aus den Ovarien heruntergelangt. Dieser «descensus» sei nicht momentan und falle zeitlich nicht mit dem Coitus zusammen, sondern könne beträchtlich von ihm differieren. Aus einer pathologisch-anatomischen Beobachtung leitet Elsholtz also Schlüsse ab, die von DE GraAf im Jahre 1673 auf Grund von Tierexperimenten bestätigt wurden. Prévost und Dumas in Genf sollten hundertfünfzig Jahre später diese Deduktion des preußischen Arztes und die mit einfachen Hilfsmitteln durchgeführten Untersuchungen DE GRAAFS auf breiterer Grundlage weiterverfolgen und zur sicheren Lehre erheben ${ }^{19}$, und in der Entdeckung des Säugetiereies durch KARL ERnst von BAER (1827) erhielt dieses theoretische Postulat, das B. der Allgemeinheit als Erster bekanntgegeben hatte, die endgültige faktische Begründung.

d) Eierstock

Neben einigen unzweifelhaften Dermoiden, die aus den Werken von Lazarus Rivière (1646), Ysbrand van Diemerbroek (1672) und Fabricius Hildanus im Sepulchretum beschrieben sind ${ }^{20}$, und einigen als Ovarialsarkomen zu bewertenden krankhaften Veränderungen, die DE GRAAF veröffentlicht hatte, ist hier von besonderem Interesse eine maligne entartete Ovarialzyste, auf die wegen der ausführlichen Beschreibung ab-

${ }^{19}$ Vgl. dazu meine frühere Studie: The Contribution of Geneva Physicians to the Physiology of Development in the 19th Century, Bull. Hist. Med. 21, 871-97 (1947), und die dort angegebenen Quellen.

${ }^{20}$ Hildanus hielt einen derartigen Tumor für eine Geschwulst des Uterus. 
schließend noch kurz eingegangen sei. Wir verdanken die Krankengeschichte dem Basler Anatomen Caspar BauHin (1560-1624). ${ }^{21}$

Es handelt sich um die Frau eines Vornehmen, die 1588 nach 16jährigem Leiden starb, wobei es nach «Tertianfieber», Krämpfen und stechenden Schmerzen zu Abgang von Eiter aus Scheide und After gekommen war. Der Arzt hatte einen großen Tumor rechts sowie allgemeine Schwellung des Bauches und der Unterschenkel konstatiert.

Die von BAuHIN persönlich ausgeführte Autopsie ergab folgendes: Der durch Paracentese eröffnete Tumor enthielt 9 Pfund Flüssigkeit (2-3 Basler Maße), ein zweiter, vorerst nicht näher untersuchter Tumor befand sich auf der rechten Seite, der Darm war in ganzer Länge völlig intakt, ebenso das Mesenterium. Der rechtsseitige Tumor war von einer fettigen Hülle umkleidet, an verschiedenen Stellen mit dem Peritoneum verwachsen, im Innern befand sich ein zweiter, nach außen verwachsener Tumor und viele kleine Blasen. Übriges Genitale ohne Besonderheiten, mit Ausnahme von zwei Fisteln, die vom Collum zum Anus führten.

Wichtiger als der pathologisch-anatomische Befund scheint uns auch in diesem Falle, bei dem es sich vermutlich um ein bösartiges Cystadenoma ovarii handelte, die Methode, mittels der die verschiedenen Befunde erhoben wurden. Diese recht fortschrittlich anmutende Sektionstechnik, die im einzelnen aus den mitgeteilten Befunden abgeleitet werden kann, dürfte auch die ausführliche Wiedergabe des Berichtes von BAUHIN rechtfertigen, der sich hier über das Anatomische hinaus als äußerst gewandter Untersucher erweist. Dabei ist zu beachten, daß diese in ihrer Art sicher vereinzelt gebliebene Sektion bereits im Jahre 1588 ausgeführt wurde. Ohne Zweifel haben derartige frühe pathologisch-anatomische Beobachtungen, besonders wenn ihnen eine so sorgfältige Technik zugrunde lag, wesentlich zur Förderung dieses neuen Zweiges der Forschung beigetragen. Daß Basel namentlich in der Gestalt von Felix Platter (1536-1614) einen gewichtigen Pionier der Pathologischen Anatomie hervorbrachte, wird an anderer Stelle ausführlicher zu zeigen $\operatorname{sein}^{22}$.

\section{Die medizingeschichtliche Beurteilung des «Sepulchretum»}

Eine kritische Würdigung des Lebenswerkes von B. wird sich heute auf das Sepulchretum beschränken dürfen. Denn obgleich der Genfer Arzt meh-

${ }^{21}$ Im Anhang der Gynaecia, die B. als Quelle angibt, ließ sich allerdings dieser Bericht nicht finden (in keiner der Ausgaben).

${ }^{22}$ Vgl. dazu auch den kleinen Beitrag zur Festschrift von Alfred LabHard, Gynäkologie und Geburtshilfe bei Felix Platter, Schweiz. med. Wschr. 71, 55 ff. (1941). 
rere weitere Sammelwerke herausgegeben hat $^{23}$, so halten sich diese doch ganz im Rahmen der entsprechenden nosographischen Literatur jener Zeit, die ihr Verfasser ja selber in scharfen Gegensatz zum ersten und wichtigsten seiner Bücher gestellt hat. Von ihm konnte er sagen, daß es einen neuen Weg eingeschlagen habe. Inwiefern bestand nun diese Ansicht B.s zu Recht? Um diese Frage zu beantworten, wird man versuchen müssen, den allgemeinen Charakter des Werkes in formaler Hinsicht und nach seinem Inhalt aus jener Zeit heraus zu verstehen, wobei es hier nicht möglich ist, die großen Linien der Heilkunde des Barocks in die Betrachtung miteinzubeziehen ${ }^{24}$.

Was zunächst die Art der Darstellung betrifft, so hat sich B. einer getreuen, meist wörtlichen Wiedergabe der Quellen befleißigt; unter Vermeidung alles Nebensächlichen gibt er in prägnanter Klarheit, oft in beabsichtigter Schematik, die früheren Texte wieder, wobei Wesentliches besonders hervorgehoben wird. Demgegenüber bleibt er in den «Scholien» dem alten Vorbild noch stark verhaftet, was auch in den häufigen Zitaten aus alten Autoren zum Ausdruck kommt. Insbesondere durch diesen «wissenschaftlichen Apparat» wird das Buch an vielen Stellen unübersichtlich, in derselben Weise wirkt sich die oft unklare Abgrenzung der einzelnen Quellen aus, besonders wenn sie von ganz verschiedenem Wert sind. Daß manche Auszüge bibliographisch unrichtig oder ungenau zitiert sind, kann man B. nicht übelnehmen, wenn man den von ihm selber dargelegten Umständen Rechnung trägt.

Dem heutigen Leser macht es große Mühe zu entscheiden, ob B. seinem Werk eigene Beobachtungen an der Leiche beigefügt hat. Ich möchte nicht wagen, aus den paar fraglichen Stellen den Schluß zu ziehen, daß dies tatsächlich der Fall ist. Jedenfalls hat er sich darüber nicht eindeutig ausgesprochen, so daß der Vorwurf Hirschs berechtigt ist, B. habe sich natürlich abgesehen von Zusätzen klinischer Art - ausschließlich literarischreproduktiv an seinem Werk betätigt. Es ist uns auch nicht gelungen, in bezug auf Befunde an der Leiche ein irgendwie ins Gewicht fallendes eigenes Urteil zu registrieren, abgesehen von den grundsätzlichen Bemerkungen im Vorwort.

${ }^{23}$ Sie sind vollständig erfaßt bei LÉon GAUTIER, La médecine à Genève jusqu'à la fin $d u$ dixhuitième siècle, Genf 1906, S. 511-3.

24 Es gilt für sie im wesentlichen immer noch das Gesamtbild, das HeNry E. Sigerist in seinem Leipziger Vortrag William Harveys Stellung in der europäischen Geistesgeschichte (Arch. Kulturgesch. 19, 158-68, 1929) in knappen Zügen entworfen hat, sowie die ausgezeichnete Einleitung von Hans Frscher in seiner Monographie über J.J.WEPFER (Zürich 1931). 
Daß von einer kausal-genetischen Betrachtungsweise, die der theoretischen Medizin seit Virchow ihr Gepräge verleiht, bei B. nicht die Rede sein kann, versteht sich von selbst.

Doch überwiegen gegenüber diesen Nachteilen die positiven Seiten des Werkes bei weitem. Die souveräne Beherrschung der damaligen Literatur ließ B. überall mit geübtem Blick die wichtigen Mitteilungen seiner Zeitgenossen herausfinden, und den Reichtum an ganz verschiedenartigen Einzelheiten dürfte auch der vorhergehende bescheidene Ausschnitt aus der Gynäkologie gezeigt haben. Wenn auch B. nicht besonders auf die Methode der Leichenuntersuchung einging, so läßt sich diese doch ableiten aus den einzelnen Befunden. Der Genfer Gelehrte hielt sich eben überall innerhalb der ihm durch seine ausschließlich praktische Betätigung gezogenen Grenzen. Aber um so verdienstvoller ist es, daß er in der Heranziehung von einschlägigen Beobachtungen weder örtliche noch zeitliche Grenzen kannte. Für unseren heutigen Geschmack mag das Sepulchretum noch viel zuviel Klinisches enthalten, aber einen Krankheitsfall, bei dem der Leichenbefund fehlt, würde man vergebens suchen. Und in diesem einen Bestreben, die klinische und die Leichen-Untersuchung unter einen Hut zu bringen, liegt eben der große Wert des Buches.

Dieser Umstand war auch für den Schöpfer der pathologischen Anatomie, G. B. Mongagni (1682-1771), ausschlaggebend, als er das Sepulchretum zur literarischen Grundlage seines eigenen Lebenswerkes machte. Der italienische Arzt schreibt im Vorwort zu seinem berühmten Buch, daß das Verdienst B.s hoch anzuschlagen sei, indem er die früheren verstreuten Beobachtungen, von denen niemand etwas gewußt habe, gesammelt habe. Ursprünglich scheint MorgagNI, wie er im Vorwort selber mitteilt, «in jugendlicher Begeisterung» die Absicht gehabt zu haben, das bei B. Fehlende und als unzweckmäßig Empfundene in einer von ihm bearbeiteten Neuausgabe zu verbessern. Trotz den Mängeln des Sepulchretum, die MoRGaGNI Punkt für Punkt bespricht, wisse er jedoch kein Werk, das wegen seines Nutzens so sehr eine Neubearbeitung verdiente wie gerade jenes. Auch im Text des in Padua entstandenen Werkes begegnet uns häufig der Name des Genfer Gelehrten, der übrigens ständig als «BonNET» geschrieben wird ${ }^{25}$. Auch in seiner Einteilung will MorgagnI nach seiner Äußerung im Vorwort dem Vorbild B.s gefolgt sein, wie er denn in Verbesserungs-

25 Theophil Bonet scheint mit dem berühmten Biologen und Philosophen Charles BoNNET (1720-1793) in keinen verwandtschaftlichen Beziehungen zu stehen. 
vorschlägen usw. überhaupt immer wieder auf das Genfer Werk zurückkommt (besonders im Zusammenhang mit dem Register). Es ist also sicher nicht übertrieben, wenn man B. als den bei der Abfassung des ersten Handbuches der Pathologischen Anatomie allzeit gegenwärtigen «spiritus rector» betrachtet, ohne den dieses undenkbar wäre.

Auch im Urteil der beiden großen medizinischen Enzyklopädisten des 18. Jahrhunderts nimmt B. eine hervorragende Stellung ein. Hermann BoerhaAve (1668-1738), der einflußreiche Leidener Gelehrte, schreibt bekanntlich in seinem Methodus studii medici $(1751)^{26}$, es sei kaum ein Buch nützlicher als das Sepulchretum, das mehr Licht in die Erforschung der Krankheiten gebracht habe als die Theorien aller zeitgenössischen Ärzte. Und im Lob Albrecht von Hallers (1708-1777)27, der das Genfer Buch als «Immortale opus, quod solum pro pathologica bibliotheca sit» bezeichnet und B. einen «industrius collector neque propriis destitutus adnotationibus» nennt, kommt die Wertschätzung eines Mannes zum Ausdruck, der wie kein zweiter das gesamte Schaffen der Jahrhunderte vor ihm überblickte.

\section{Quellen-Hinweise und wichtigste Literatur}

\section{Gedruckte Quellen und Darstellungen}

1. a) Sepulchretum sive Anatomia practica ex cadaveribus morbo denatis, proponens historias et observationes omnium corporis humani affectuum, ipsorumque causas reconditas relevans, Genevae (Sumptibus Leonardi Chouet) 1679.

b) 2. Auflage: Editio altera, quam novis commentariis et observationibus innumeris illustravit, ac tertia ad minimum parte auctiorem fecit Johannes Jacobus Manget . . L Lugduni (Sumptibus Cramer et Perachon) 1700.

2. (EDOUard) Cornaz,

a) Un chirurgien d'outre-tombe, Musée Neuchâtelois vol. 2, 1865, p. 85-95 (Forts. p. 101-10, 131-42, 161-9).

b) Notices relatives à l'histoire médicale de Neuchâtel: $V$. Une préface de THÉopHILE Bonet. Musée Neuch. 7, 1870, p. 289-90.

3. Jean Senebier, Histoire littéraire de Genève, Genève 1786 (tom. II, p. 226; Bibliographie: p. 227/8).

4. J.-A. GALIFFe, Notices généalogiques, Genève 1836 (t. III, p. 64-7).

${ }^{26}$ Methodus studii medici, Amsterdam 1751, Bd. 2, S. 618. Hoc opere vix aliud utilius est, magisve meretur suppleri, et ad nostra tempora deduci, ex quo certe major lux ad veras morborum sedes et causas eruendas adfulget, quam ex omnibus aliorum ad ea tempora medicorum theoriis.

${ }^{27}$ Bibliotheca medicinae practicae, Bern 1779, Bd. 3, S. 236-8. 
5. La France protestante (Eugène et Emile HaAg). $2^{\mathrm{e}}$ éd. Paris 1879. Art. Bonnet: vol. 2, p. 844-7. (Die Bemerkungen zu den einzelnen Werken sind Haller entnommen.)

6. LÉon GaUtier, La médecine à Genève jusqu'à fin du $18^{e}$ siècle, Genève 1906 (p. 251-3; Bibliographie p. 511-3; Bildnis von BoNET zu S. 250).

7. Chaussier und Adelon, Biographie universelle, vol. 5, Paris und Leipzig 1854 ff., p. 30/1. (Enthält wertvolle Hinweise auf sonst nicht Bekanntes.)

8. John Viénot, Histoire du Pays de Montbéliard, Audincourt 1904.

\section{Ungedruckte Quellen}

1. Staatsarchiv Genf (Auszüge):

a) Actes notariés 1658-1689.

b) Registres du conseil 1671-1689.

2. Universitäts-Bibliothek Basel:

Die Handschriftenabteilung enthält nach der freundlichen Mitteilung von Dr. MAX Burckhardt von Theophil Bonet nur vier Briefe an Johann Caspar Bauhin (1606 bis 1685), die jedoch wenig Biographisches bieten (im Sammelband Mscr. G ${ }^{2}$ I 10, der die Briefe an BAUHIN enthält).

\section{Gerolamo Fracastoro e la dottrina del contagium vivum Di Arturo Castiglioni, Milano}

Henry E. Sigerist nell'Introduzione a quello splendido volume che costituisce il principio e il fondamento della sua «History of Medicine» recentemente pubblicato, espone con eloquente chiarezza il programma che lo storico della medicina deve seguire, e al quale egli si propone di attenersi in questa sua opera che sarà certamente il testo classico di questa disciplina nel nostro secolo. Egli scrive: «Noi dobbiamo studiare i sistemi magici, religiosi, filosofici, filologici e scientifici della medicina: non solo dunque le azioni del medico, ma le idee che lo hanno guidato. Non vogliamo investigare l'arte della medicina, ma la sua dottrina, non la pratica medica, ma la scienza medica ... Le dottrine mediche hanno sempre rappresentato un aspetto della civiltà di un dato periodo e per comprenderle pienamente devono essere familiari le altre manifestazioni di questa civiltà, la sua filosofia, la letteratura, l'arte e la musica. Noi presto troveremo che un comune denominatore, una tendenza generale o uno stile si possono riscontrare in tutte queste manifestazioni» e parlando delle grandi scoperte di 